[Technical Paper]

\title{
Effect of Current Heating on Accurate Measurements of AC Shunt Resistors at DC to $3 \mathrm{kHz}$
}

\author{
Seitaro Kon and Tatsuji Yamada \\ National Metrology Institute of Japan, National Institute of Advanced Industrial Science and Technology, Tsukuba, Ibaraki 305-8563, Japan
}

(Received July 31, 2015; accepted December 4, 2015)

\begin{abstract}
To propose a structure of high-accuracy shunt resistors, the frequency and current dependencies of ac shunt resistors with different rated current are evaluated both of ac resistance and phase angle. To evaluate these characteristics, modified current-bridge method was developed. The ac resistances of the shunts do not vary considerably with the frequency, up to $3 \mathrm{kHz}$. The phase angle of ac shunts varies proportionally to the frequency. The current dependence of the ac resistance of shunts seems to change with current value. The current dependence of the phase angle of the shunt remain stable up to $10 \mathrm{~A}$.
\end{abstract}

Keywords: Shunt Resistor, Phase Angle, Frequency Characteristic, Current Dependence, Measurement

\section{Introduction}

AC shunt resistors play an important role in several research areas where accurate current measurements are necessary, because ac shunt resistors allow for the most precise measurement of current. Therefore, for example, the evaluation of a new current sensor requires an accurate and stable current standard such as a magnetic bridge current sensor[1] and an electronic current transformer.[2] In addition, a precision ac shunt resistor is an important component of sinusoidal and non-sinusoidal power standards and accurate current measurements. For these situations, an ac shunt calibration system has been developed and measurement results for both the ac resistance and phase angle were verified up to $1 \mathrm{kHz}$.[3, 4] However, expansion of the measurement frequency and current ranges are needed for the evaluation of harmonic characteristics and high-frequency applications.[5] This paper describes the modified current-bridge method and its measurement uncertainties of the frequency range up to $3 \mathrm{kHz}$ and the current dependencies of shunt resistors for both ac resistance and phase angle.[6]

\section{Shunt Resistor}

The cage-type shunt resistors[7] were used in this study. Specifically, Fluke A40B shunt resistors with nominal values of $0.8 \Omega, 0.16 \Omega$ and $0.08 \Omega$ and a shunt resistor consisting of $10010-\Omega$ metal-foil resistors connected in paral- lel[8] with a nominal value of $0.1 \Omega$ are used. The rated current values of each shunt are $1 \mathrm{~A}, 5 \mathrm{~A}$, and $10 \mathrm{~A}$. The parallel configuration reduces the harmful effects of inductance and heat due to the input current on the resistance. The basic concept of the four shunts is the same, but the size and shape of the three shunts are slightly different from each other. According to the simple equivalent circuit, the in-phase component of the ac shunt resistor is proportional to the square of the frequency, and the

\section{Shunt $(0.08 \Omega)$}

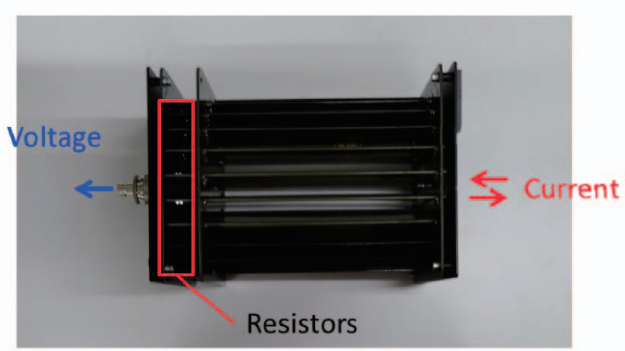

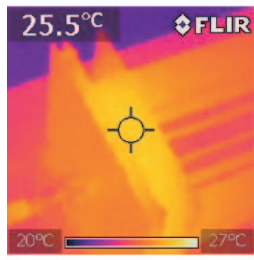

$1 \mathrm{~A}$

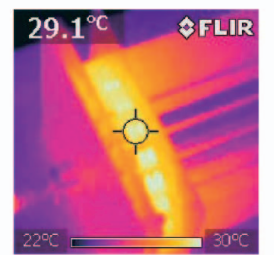

$5 \mathrm{~A}$

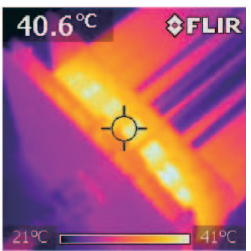

$10 \mathrm{~A}$
Fig. 1 Pictures of shunt resistors and surface temperature of a resistance element of shunt with nominal value of $0.08 \Omega$. 


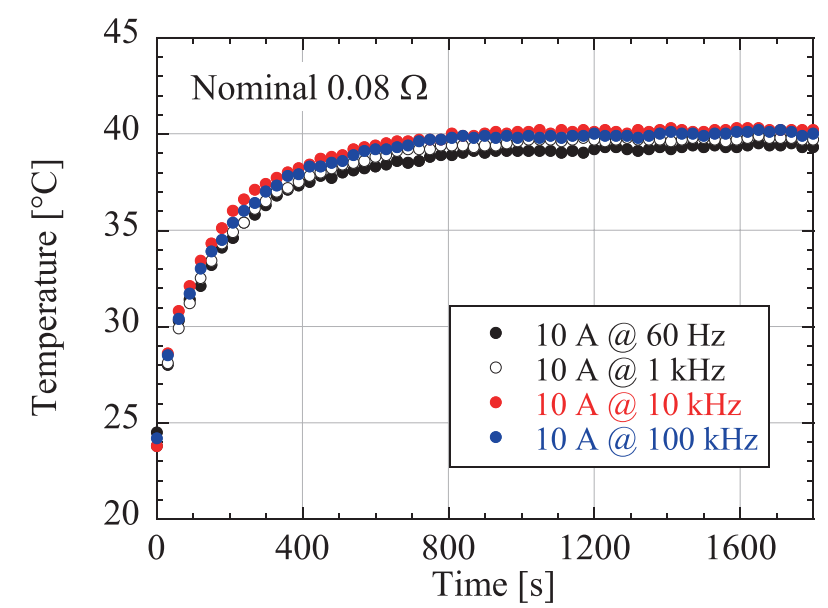

Fig. 2 Variation in surface temperature of a shunt resistance element of shunt with nominal value of $0.08 \Omega$.

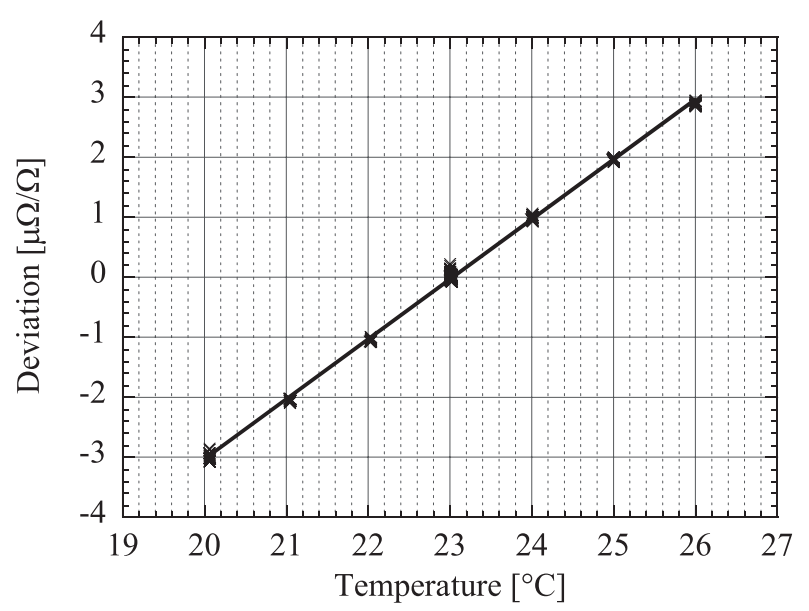

Fig. 3 Temperature dependence of the shunt resistor at dc. These values are deviations from the resistance value at $23^{\circ} \mathrm{C}$.

quadrature component is proportional to the frequency.[4]

Figure 1 shows the relationship between input current and variations of surface temperatures of a resistance element of the shunt resistor with nominal value of $0.08 \Omega$ measured by thermography at input currents of $1 \mathrm{~A}, 5 \mathrm{~A}$, and $10 \mathrm{~A}$ and an ambient temperature of $23^{\circ} \mathrm{C} \pm 1^{\circ} \mathrm{C}$. The temperature distribution is not a uniform state because the main heat sources are resistors connected in parallel. The temperature stabilized after $60 \mathrm{~min}$ from the current input because of a thermal equilibrium. The temperature of the resistor increased to $27^{\circ} \mathrm{C}$ at $1 \mathrm{~A}, 30^{\circ} \mathrm{C}$ at $5 \mathrm{~A}$, and $41^{\circ} \mathrm{C}$ at $10 \mathrm{~A}$. A waiting time derived from a thermal equilibrium of at least $60 \mathrm{~min}$ is necessary for accurate measurement of ac shunt resistors as shown in Fig. 2. These values did not depend on the frequency of input current.

Figure 3 shows the temperature dependence of the shunt resistor with nominal value of $0.1 \Omega$ at dc measured by dc resistance standards. [9] As you can see, the temperature coefficient of the shunt resistor is about $+1 \mathrm{ppm} /{ }^{\circ} \mathrm{C}$.

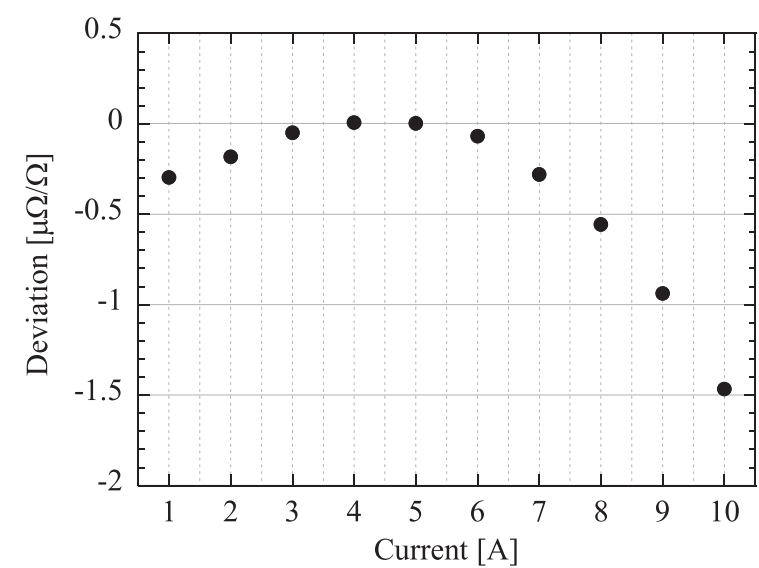

Fig. 4 Current dependence of the shunt resistor at dc. These values are deviations from the dc resistance value at $5 \mathrm{~A}$.

Furthermore, as shown in Fig. 4, the current dependence of the shunt resistor with nominal value of $0.1 \Omega$ at $\mathrm{dc}$ was measured. The deviations from the value at $5 \mathrm{~A}$ are less than $2 \mu \Omega / \Omega$ with the current value between $1 \mathrm{~A}$ and $10 \mathrm{~A}$.

\section{Measurement Method}

\subsection{Modified current-bridge method}

The current bridge method has been developed for accurate measurement of shunt resistors and measurement results for both the ac resistance and phase angle of shunt resistors were verified up to $1 \mathrm{kHz}$.[3,4]

The principle of current-bridge method is to compare input current with a current derived from the output of the shunt resistor. The current derived from the shunt resistor is produced with a buffer amplifier (BUF) and a calibrated $1 \mathrm{k} \Omega$ ac standard resistor. The buffer amplifier is evaluated by comparing the input and output with the switching system that enables signal-path compensation in advance. The current-bridge method uses a current comparator (CC) and current transformer (CT). A CT that is also calibrated by the ac current ratio standard[10] is used for setting the appropriate current ratio of the two currents to be compared. For example, a CT of ratio 100 and a CC of ratio 100 are used when the shunt with nominal value of $0.1 \Omega$ is evaluated. A compensation current source has been fabricated with careful attention paid to prevent any ground loops. It generates in-phase and quadrature currents.

Then, the impedance of the shunt $Z_{x}$ is defined by

$$
Z_{x}=R_{x}\left(1+\varepsilon+j \theta_{x}\right)
$$

$Z_{x}$ is written with the appropriate approximations by using current-bridge method 


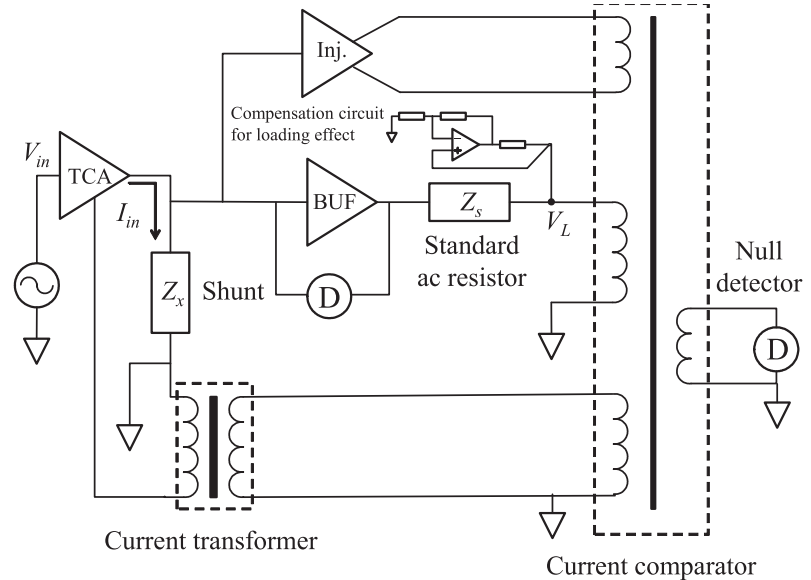

Fig. 5 Schematic of modified current-bridge method for evaluation of shunt resistors.

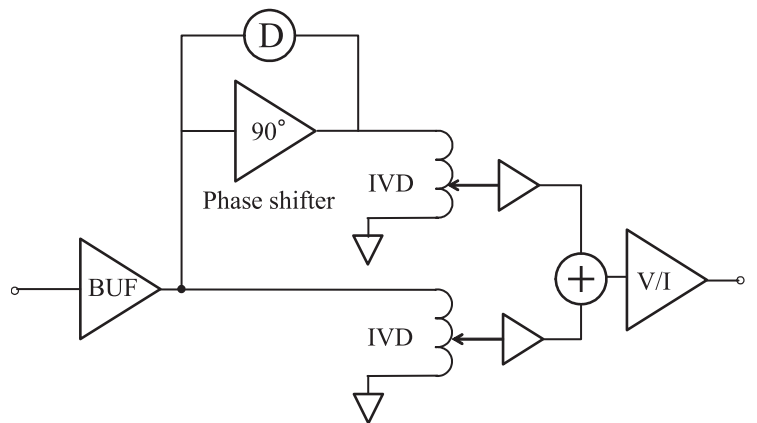

Fig. 6 Adjustable current source for injecting error current to current comparator (Inj. in Fig. 5).

$$
Z_{x}=\frac{R_{s} N_{C T}}{n_{c c}}\left(1+\varepsilon_{C T}+\varepsilon_{R}-\varepsilon_{\alpha}-\varepsilon_{c c}-\varepsilon_{\beta}-\frac{R_{s} \gamma}{n_{c c}} \varepsilon_{\gamma}-\frac{R_{s} \gamma}{n_{c c}} X\right)
$$

where $R_{s}, N_{C T}, n_{c c}$, and $\gamma$ are the nominal value of the standard ac resistor, the ratio of the current transformer, the ratio of the current comparator, and the voltage-current conversion rate of the error current source, respectively; and $\varepsilon_{C T}, \varepsilon_{R}, \varepsilon_{\alpha}, \varepsilon_{C C}, \varepsilon_{\beta}, \varepsilon_{\gamma}$, and $X$ are the respective errors of the current transformer, ac resistor, buffer amplifier, current comparator, compensation network, and error current source, and the measurement value. [3, 4]

However, the measurement system that uses current bridge method had the limitation of the measurement frequency range because of the leak current due to the stray capacitance. However, the expansion of the measurement frequency and current ranges are needed. Therefore, the current-bridge method is modified.

The basic concept of the modified current bridge method is the same with current-bridge method. However, the defining point of the signal is different and therefore the circuit configurations are also different. Modified cur- rent-bridge method can reduce the leak current due to the stray capacitance and the effect of the ground-loop.

Figure 5 shows the schematic diagram of the modifiedcurrent bridge method. The modified current-bridge method uses a current comparator and current transformer. An input current $I_{\text {in }}$ is compared with the current derived from the output voltage of a shunt resistor that is produced with a buffer amplifier and calibrated ac standard resistor with nominal value of $1 \mathrm{k} \Omega$. A current transformer is also calibrated by the ac current ratio standard. [10] It is used for setting the appropriate current ratio of the two currents to be compared by using current comparator.

An error current injection (Inj. in Fig. 5) for the current comparator consists of inductive voltage dividers (IVDs),[11] a quadrature phase shifter based on the adjustable active all-path filter, and a voltage-to-current converter as shown in Fig. 6. The adjustable current source generates in-phase and quadrature currents corresponding to the settings of the IVDs.

The phase shifter is adjusted by using 6-decade adjustable resistor and a null detector for keeping 90 degree phase difference between in-phase and quadrature currents at each measurement completely. A compensation circuit for the loading effect compensates for the current deviation experienced from the winding impedance of the current comparator. The compensation circuit senses the voltage $V_{L}$ and automatically provides the appropriate current until $V_{L}$ is equal to zero.

Finally, we can get both in-phase and quadrature components of ac shunt resistors in the level of $10^{-7}$ with measurement uncertainty by using this method.

\subsection{Uncertainty}

The combined standard uncertainty is obtained by appropriately combining the standard uncertainties of all contribution factors.

Table 1 shows the uncertainty budget of the modified current-bridge method at the frequencies of $55 \mathrm{~Hz}$ to 3 $\mathrm{kHz}$. The uncertainty budget does not change with the current value between $1 \mathrm{~A}$ and $10 \mathrm{~A}$.

The standard uncertainties of the ratio error of the current transformer and current comparator are calibrated by the ac current ratio standard. The standard uncertainty of the resistance and phase angle of the reference standard ac resistor is evaluated at $1 \mathrm{kHz}$ by an ac resistor standard that is related to the quantized Hall resistance standard.[9] The effects derived from the difference between the calibration frequency of the standard ac resistor and the actual 
Table 1 Uncertainty budget of the modified current-bridge method for at $55 \mathrm{~Hz}, 400 \mathrm{~Hz}, 1 \mathrm{kHz}$, and $3 \mathrm{kHz}$ at $5 \mathrm{~A}$.

\begin{tabular}{|c|c|c|c|c|c|c|c|c|}
\hline \multirow[t]{2}{*}{ Source of uncertainty } & \multicolumn{4}{|c|}{$\begin{array}{c}\text { Relative Standard uncertainty } \\
\text { (in-phase) } \\
{[\mu \Omega / \Omega]}\end{array}$} & \multicolumn{4}{|c|}{$\begin{array}{c}\text { Standard uncertainty } \\
\text { (quadrature) } \\
{[\mu \mathrm{rad}]}\end{array}$} \\
\hline & $55 \mathrm{~Hz}$ & $400 \mathrm{~Hz}$ & $1 \mathrm{kHz}$ & $3 \mathrm{kHz}$ & $55 \mathrm{~Hz}$ & $400 \mathrm{~Hz}$ & $1 \mathrm{kHz}$ & $3 \mathrm{kHz}$ \\
\hline Current transformer & 0.6 & 0.6 & 1.8 & 20 & 0.9 & 3.9 & 9.5 & 29 \\
\hline Standard ac resistor & 0.3 & 0.3 & 0.3 & 0.3 & 4 & 4 & 3.8 & 3.8 \\
\hline Frequency dependence of ac resistor & 0.5 & 0.5 & 0.0 & 0.8 & 0.7 & 0.7 & 0.0 & 12 \\
\hline Current dependence of ac resistor & 0.8 & 0.8 & 0.8 & 0.8 & 0.2 & 0.2 & 0.2 & 0.2 \\
\hline Buffer amplifier & 1.2 & 1.2 & 1.2 & 1.2 & 1.2 & 1.2 & 2.3 & 5.8 \\
\hline Current comparator & 0.6 & 0.6 & 0.7 & 20 & 0.9 & 1.0 & 9.5 & 29 \\
\hline Compensation circuit for the loading effect & 0.2 & 0.2 & 1.0 & 3.0 & 0.2 & 0.2 & 1.0 & 3.0 \\
\hline Error current injection & 0.2 & 0.2 & 0.8 & 3.0 & 1.1 & 1.1 & 2.1 & 3.7 \\
\hline Measurements & 1.0 & 1.0 & 1.0 & 1.0 & 1.0 & 1.0 & 1.0 & 1.0 \\
\hline Combined standard uncertainty & 2.1 & 2.1 & 2.9 & 29 & 4.7 & 6.0 & 15 & 44 \\
\hline Expanded uncertainty $(k=2)$ & 4.2 & 4.2 & 5.8 & 58 & 9.4 & 12 & 30 & 88 \\
\hline
\end{tabular}

frequency of the measurement, for example, $1 \mathrm{kHz}$ and 3 $\mathrm{kHz}$, are considered. In addition, the effects of the current dependence of the standard ac resistor also be considered. The errors of the buffer amplifier, mainly the errors of the gain and phase, are monitored by the measurements of the voltage difference between the input voltage and the output voltage using a lock-in amplifier. The errors of the buffer amplifier are possibly load dependent. The impedance of the comparison windings of current comparator is smaller than $0.1 \Omega$. However, the comparison windings are connected to the standard ac resistor in series. Therefore, the impedance of the windings greatly influences the ac shunt resistor measurements. A compensation circuit can cancel out the effects of the impedance of the windings automatically. The standard uncertainty of a compensation circuit is estimated on the basis of the resistance errors and the reduction of the open-loop gain that depends on the frequency. The standard uncertainty of the error current injection for the current comparator is estimated by the ratio of the IVDs, and the transfer function of the phase shifter. The error of the in-phase component depends on the resistance errors of the circuit and the reduction of the gain with frequency. The error of the quadrature component mainly depends on the accuracy of the quadrature phase shifter.

The main factor contributing to the combined standard uncertainties are the uncertainties of current transformer and current comparator at $3 \mathrm{kHz}$. The measurement uncertainties of the current transformer and current com- parator increase sharply with increasing frequency and reduction of them are very important for more accurate measurements of ac shunt resistors.

\section{Results}

\subsection{Frequency characteristics}

Several ac shunt resistors with different rated currents were evaluated by the modified current-bridge method. Figure 7 shows measurement results of ac shunt resistors and a comparison of the results of the modified currentbridge method to those of the dc-resistance and ac-dc-difference methods at $1 \mathrm{~A}$ and $5 \mathrm{~A}$. The dc-resistance and acdc-difference measurements using a thermal converter easily extend the frequency range.[12, 13] However, the dc-resistance and ac-dc-difference methods cannot provide a phase angle measurement because the phase angle information is lost during the thermoelectric conversion by a thermal converter. The results are consistent with each other within the measurement uncertainties at $55 \mathrm{~Hz}$, $400 \mathrm{~Hz}$, and $1 \mathrm{kHz}$. The frequency characteristics of inphase component are flat up to $3 \mathrm{kHz}$. The results for the phase angle were proportional to the frequency for all of ac shunt resistors. The frequency responses of the phase angle of the ac shunt resistors are supposed to be proportional to the frequency in the theory of the equivalent circuit model. This means that the phase angle measurements using the modified current-bridge method are plausible so far. However, the measurement results of phase angle should be confirmed by the measurement 

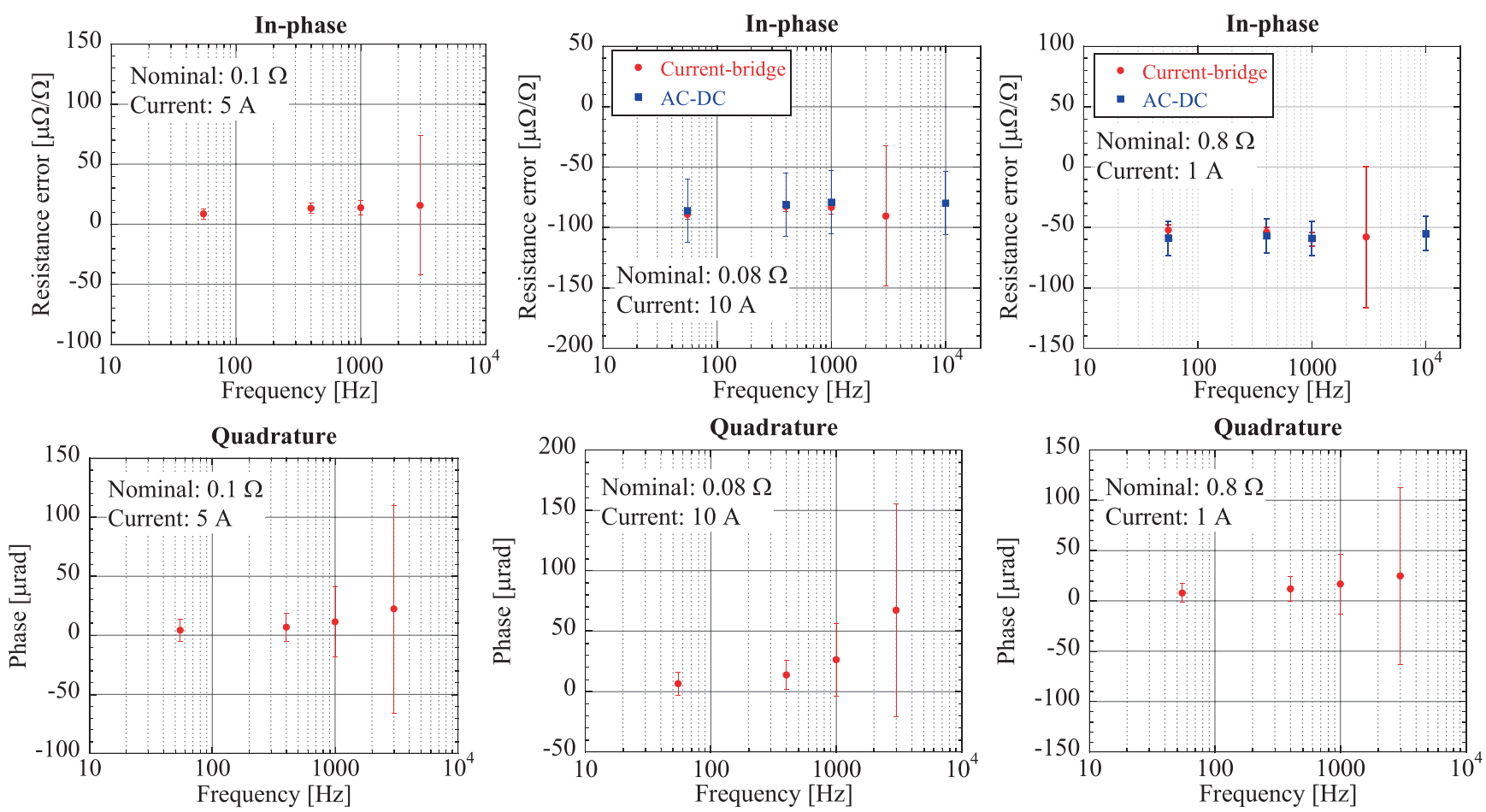

Fig. 7 Frequency characteristics of ac shunt resistors for both in-phase and quadrature components.
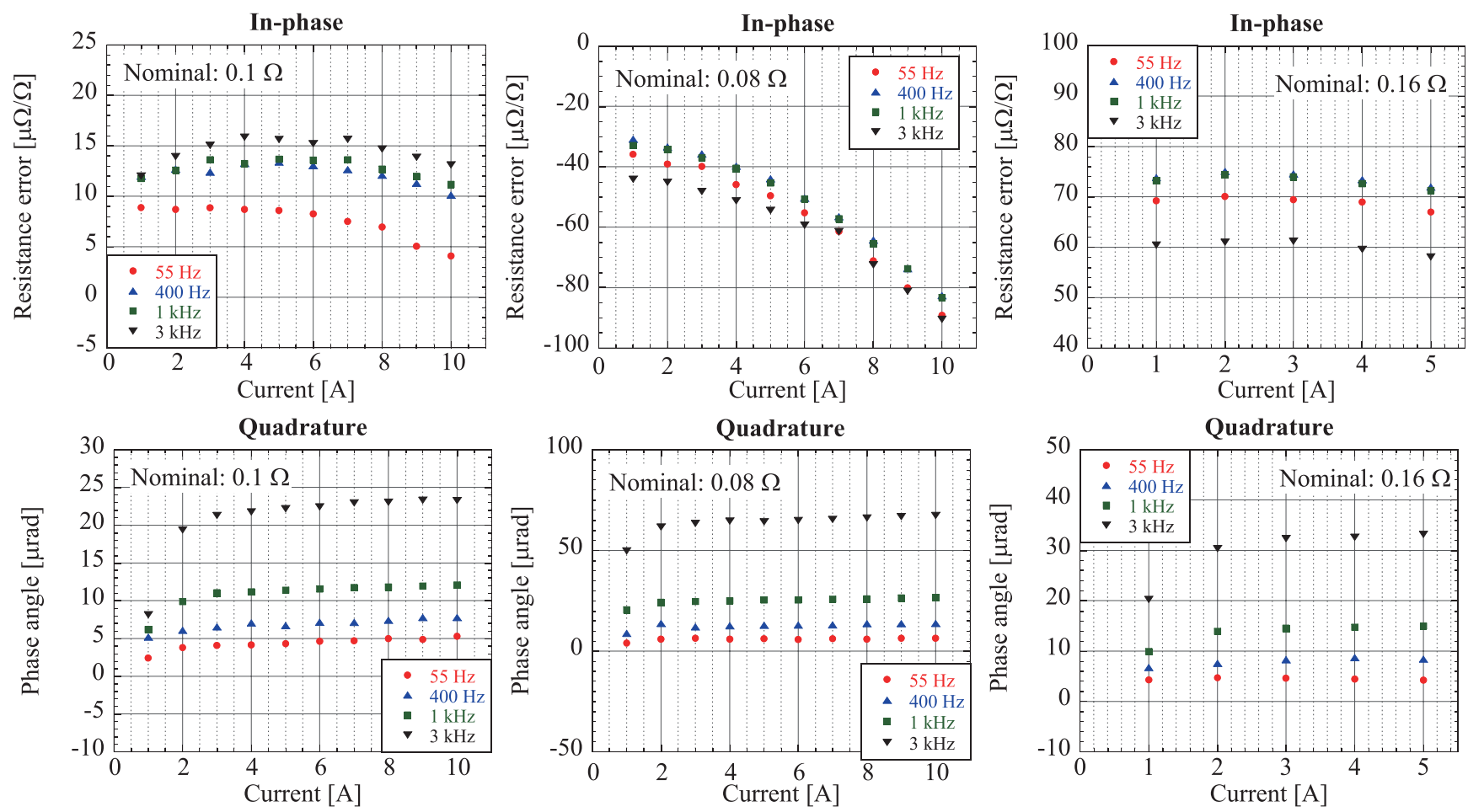

Fig. 8 Current dependence of ac shunt resistors for both in-phase and quadrature components. Measurement uncertainties of in-phase components are $4.2 \mu \Omega / \Omega$ at $55 \mathrm{~Hz}, 4.2 \mu \Omega / \Omega$ at $400 \mathrm{~Hz}, 5.8 \mu \Omega / \Omega$ at1 kHz, and $58 \mu \Omega / \Omega$ at $3 \mathrm{kHz}$.

results of other methods or international comparison.

\subsection{Current dependence}

Figure 8 shows the current dependence of the ac shunt resistors obtained by the modified current-bridge method at $55 \mathrm{~Hz}, 400 \mathrm{~Hz}, 1 \mathrm{kHz}$, and $3 \mathrm{kHz}$. The in-phase compo- nent seems to change slightly with increasing current for the ac shunt resistors with nominal values of $0.1 \Omega 0.16 \Omega$. However, the results at different currents are consistent with each other within the expanded uncertainties (coverage factor: $k=2$ ). Meanwhile, the current dependence of 


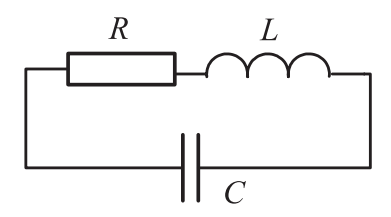

Fig. 9 Schematic of a simple equivalent circuit model of an ac shunt.

ac shunt resistors with nominal value of $0.08 \Omega$ clearly decrease with increasing current. To clarify the changes in the in-phase components of the ac shunt resistors, the measurement uncertainties of the modified current-bridge method have to be reduced.

As shown in Fig. 9, a simple equivalent circuit for ac shunt can be expressed. According to the equivalent circuit, the impedance of the ac shunt $Z$ is given by

$$
Z=\frac{R}{\left(1-\omega^{2} L C\right)^{2}+(\omega C R)^{2}}+j \frac{\omega L-\omega C R^{2}-\omega^{3} L^{2} C}{\left(1-\omega^{2} L C\right)^{2}+(\omega C R)^{2}}
$$

where $\omega$ is the frequency, $L$ and $C$ are stray impedance, and $R$ is the resistance. The resistance increase with increasing temperature. Therefore, the ac resistance of a shunt resistor decreases with increasing surface temperature of each resistor element.

Further, the current dependencies of the quadrature components remain stable for all of shunt resistors. These measurement results of current dependence did not depend on the measurement frequency.

\section{Conclution}

A modified current-bridge method for accurate measurements of ac shunt resistors has been developed, and its measurement uncertainties were estimated. To verify the method, a comparison of the measurement results obtained by different methods was performed. These comparison results showed good agreements and the quadrature components were proportional to the frequency, as in the theory of equivalent circuit model.

The current dependencies of ac shunt resistors were also evaluated by modified current-bridge method. The inphase components of ac shunt resistors seem to decrease with increasing current. However, the measurement uncertainty of modified current-bridge method should be reduced to clarify these results. The current dependencies of the quadrature components remain stable for all of shunt resistors. These current dependencies of both inphase and quadrature components of ac shunt resistors did not depend on the measurement frequency.

\section{References}

[1] T. Yamada, S. Kon, and T. Tadatsu, "Transresistance calibrations and temperature dependence evaluation of a magnetic bridge current sensor with shunt standards," Meas. Sci. Technol., Vol. 22, No. 10, p. 105204, August 2011.

[2] T. Yamada, S. Kon, N. Hashimoto, T. Yamaguchi, K. Yazawa, R. Kondo, and K. Kurosawa, "ECT evaluation by an error measurement system according to IEC 60044-8 and 61850-9-2," IEEE Trans. Power Del., Vol. 27, No. 3, pp. 1377-1384, July 2012.

[3] S. Kon and T. Yamada, "Uncertainty evaluations of an AC shunt calibration system with a load effect reduction circuit," IEEE Trans. Instrum. Meas., Vol. 60, No. 7, pp. 2286-2291, July 2011.

[4] S. Kon and T. Yamada, "AC shunt calibration using a current-bridge method and its validation,” IEEJ Trans., Vol. 9, No. 6, pp. 577-580, Nov. 2014.

[5] T. Yamada, S. Kon, N. Sakamoto, and H. Kato, "Uncertainty estimation and performance evaluation of a non-sinusoidal power measurement standard," IEEJ Trans. Fundam. Mater., Vol. 132, No. 3, pp. 251-256, March 2012.

[6] S. Kon and T. Yamada, "Effect of current heating on accurate measurements of ac shunt resistors," ICEPIAAC 2015 Proceedings, pp. 144-148, April 2015.

[7] K. Lind, T. Sørsdal, and H. Slinde, "Design, modeling, and verification of high-performance AC-DC current shunts from inexpensive components," IEEE Trans. Instrum. Meas., Vol. 57, No. 1, pp. 176-181, January 2008.

[8] T. Tsuchiyama and T. Tadokoro, "Development of a high-precision ac standard shunt for ac power measurement," 2002 CPEM Dig., pp. 254-255, June 2002.

[9] T. Oe, K. Matsuhiro, T. Itatani, S. Gorwadkar, S. Kiryu, and N. Kaneko, "Development of quantum hall array resistance standards at NMIJ," IEEE Trans. Instrum. Meas., Vol. 60, No. 7, pp. 25902595, July 2011.

[10] T. Yamada and S. Kon, "Error and uncertainty estimations for a passive-CC-based ac current ratio standard at high audio frequencies,” IEEE Trans. Instrum. Meas., Vol. 64, No. 6, pp. 1546-1552, June 2015.

[11] Y. Nakamura, "Calibration and uncertainty estimation of a two-staged inductive voltage divider," AIST Bull. Metrol., Vol. 4, No. 1, pp. 45-52, July 2005.

[12] J. R. Kinard, T. E. Lipe, and C. B. Childers, "AC-DC 
difference relationships for current shunt and thermal converter combinations," IEEE Trans. Instr. Meas., Vol. 40, No. 2, pp. 352-355, April 1991.

[13] J. R. Kinard, T. E. Lipe, and C. B. Childers, "Exten- sion of the NIST AC-DC difference calibration service for current to $100 \mathrm{kHz}$," J. Res. Natl. Inst. Stand. Technol., Vol. 102, No. 1, pp. 75-83, January-February 1997.

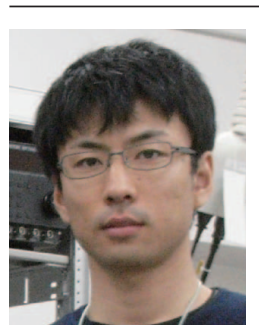

Seitaro Kon was born in Japan in 1980. He received the B.S. degree in physics from the Tokyo University of Science, Tokyo, Japan, in 2005, the M.S. degree in environmental science from Tsukuba University, Tsukuba, Japan, in 2007, and the Ph.D. degree in engineering from Shinshu University, Nagano, Japan, in 2014. He joined the National Metrology Institute of Japan, National Institute of Advanced Industrial Science and Technology (AIST), Tsukuba, in 2007, as a Research Scientist. He has been involved in highly precise current, impedance, power measurements, and the development of shunt resistor standard.

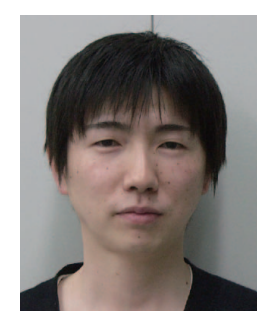

Tatsuji Yamada received the Ph.D. degree in electrical engineering from Nagoya University, Nagoya, Japan, in 2001. He was with the Electricity Division, National Institute of Standards and Technology, Gaithersburg, MD, USA, from 2001 to 2003, where he has been involved in current sensor evaluation. He joined the National Metrology Institute of Japan, National Institute of Advanced Industrial Science and Technology (AIST), Tsukuba, in 2003. His current research interests include power quality measurements, asynchronous sampling measurements, voltage ratio, and current ratio measurements. 